\title{
Die Spaltung des krystallinischen Eier- und Serumalbumins sowie des Serumglobulins durch Pepsinverdauung.
}

\author{
Von
}

Dr. F. Umber.

Volontär-Assistent der med. Klinik.

Aus dem physiologisch-chemischen Institut zu Strassburg. Neue Folge Nr. 7.) (Der Redaction zugegangen am 12. Mai 1898.)

Das grosse Eiweissmolekül wird durch die peptische Spaltung in eine verhältnissmässig geringe Anzahl von Bruchstücken zerlegt, welche selbst weiterhin nicht mehr gespalten werden. Diese Spaltungsmethode hat daher vor allen tiefer eingreifenden, wie z. B. Zersetzung mit Alkali, Säure oder Trypsin, den grossen Vorzug, dass sie uns eine klare Uebersicht über die wenigen ersten Bruchstücke ermöglicht. Freilich sind diese immer noch complicirte Verbindungen von hohem Molekulargewicht und somit einem Verständniss ihres Aufbaues noch wenig zugänglich. Trotzdem erscheint eine Bearbeitung des Eiweissproblems von dieser Seite aussichtsvoll. Die vielfachen Versuche, welche auf die Feststellung der letzten Spaltungsprodukte der Eiweissstoffe hinzielten, haben bisher keinen Einblick in den Bau des Eiweissmoleküls gewährt, weil einerseits ihre grosse Anzahl, andererseits die Unmöglichkeit, sie einzeln quantitativ zu . bestimmen, einer ausreichend begründeten Vorstellung über ihre gegenseitige Verknüpfung im Wege steht. Dagegen dürften wir wohl durch Zerlegung der ursprünglichen grossen Eiweissmoleküle in Complexe, welche ihrerseits in mindercomplicirte Zwischenstufen, schliesslich in Endprodukte zerspalten werden können, der erwünschten Aufklärung näher kommen.

Diesen Weg einzuschlagen, erschien solange nicht beson- 
ders vielversprechend, als die peptischen Spaltungsprodukte zumeist nur ihren Gattungsmerkmalen nach bekannt waren, wie sie von Kühne und seinen Schülern, namentlich $\mathrm{Neu-}$ meister, aufgestellt worden sind. Darnach unterschied man primäre, secundäre Albumosen und Peptone. Die sorgfältigen und mühevollen Untersuchungen'Kühne's, ebenso Chittenden's und ihrer Schüler haben an den untersuchten Eiweisskörpern im Allgemeinen nur diese Stufen sicherstellen und die so erhaltenen, nicht ohne Schwierigkeit gereinigten Produkte zur Analyse bringen können. Finden sich auch bei den Spaltprodukten verschiedener Abstammung ähnliche, nur zuweilen etwas verschiedene Eigenschaften, so gestatten sie doch kaum einen Rückschluss auf den Bau des zerfallenen Urmoleküls; denn abgesehen von den primären Albumosen, von denen zumal die Protoalbumose eine einheitliche Substanz vorzustellen scheint, hatte man es, wie schon nach ihrem Verhalten zum Trypsin zu schliessen war, mit Gemengen zu thun, deren Trennung unerlässlich war.

Vor Kurzem erbrachte E. P. Pick ${ }^{1}$ ) den Nachweis, dass das Gemenge der sogenannten Deuteroalbumosen zum Mindesten aus drei, dasjenige der Peptone zum Mindesten aus zwei Individuen besteht, eine Erfahrung, welche zu neuerlichen Versuchen einladen musste. Zunächst war festzustellen, ob diese Vielheit der peptischen Spaltprodukte sich in gleicher Weise auch bei anderen Proteinstoffen vorfindet. Von vornherein liess sich ja vermuthen, dass einfachere Eiweisskörper als Fibrin eine geringere, complicirtere, dagegen eine grössere Zahl solcher grossmolekularer Bruchstücke liefern würden. Sodann waren die Erfahrungen Pick's an Eiweisskörpern zu prüfen, welche eine grössere Gewähr für ihre Einheitlichkeit bieten, als das im Allgemeinen recht unreine Fibrin. In dieser Beziehung können nur die Untersuchungen der krystallinischen Phytovitelline von Neumeister ${ }^{2}$, von Chittenden und

1) E. P. Pick, Ein neues Verfahren zur Trennung von Albumosen und Peptonen. Zeitschr. f. physiol. Chemie, Bd. XXIV, S. 246.

2) Neumeister, Ueber Vitellosen, Zeitschr. f. Biol., Bd. 23, 1887. 
Hartwell ${ }^{1}$ ) und von Chittenden und L. B. Mendel ${ }^{2}$ ) als einwurfsfrei gelten. Nicht dasselbe kann vom Eieralbumin gesagt werden, das Chittenden und Bolton ${ }^{3}$ ) sowie Contejean ${ }^{4}$ ) mit Pepsin spalteten. Die beiden Ersteren haben das Eiweiss zum Theil durch Magnesiasulfat, zum Theil durch verdünnte Essigsäure, Contejean nur durch letztere nach Möglichkeit globulinfrei gemacht. Nun ist jedoch einerseits die Globulinabscheidung auf letzterem Weg erwiesenermassen eine höchst unvollkommene, andererseits enthält das noch übrigbleibende Albumingemenge zum Mindesten zwei Eiweissstoffe, nämlich das krystallisirende Albumin, sowie das bisher nicht krystallinisch erhaltene, schwefel- und stickstoffreichere Albumin: Auch das Globulin, das Neumeister ${ }^{5}$ ) verarbeitete, musste mit dem fibrinogenähnlichen Körper, der auch nach Abscheidung des Fibrins im Serum verbleibt - Hammarsten's Fibringlobulin verunreinigt sein. Das Myosin, das Kühne und Chit tenden ${ }^{6}$ ) nach den Vorschriften von Danilewsky dargestellt haben, ist nach den Erfahrungen v. Fürth's ${ }^{7}$ ) als ein Gemenge von Myosinfibrin und Myogenfibrin und nur zum geringsten Theil als Myosin anzusehen. Ob ferner das successive mit Wasser, Alkali, Säure, Alkohol und Aether extrahirte Nackenband, wie es Chittenden und $\mathrm{Hart}^{8}$ ) in ihren Verdauungsversuchen über Elastin benutzten, einen homogenen Körper darstellt, ist zum Mindesten fraglich.

1) Chittenden und Hartwell, Krystallinisches Globulin und Globulosen oder Vitellosen, Journal of physiology, Bd. 11, 1890.

2) Chittenden und L. B. Mendel, Ueber die Proteolyse von krystallisirtem Globulin, Journ. of physiol., Bd. 17, 1895.

3) Chittenden und Bolton, Eieralbumin und Albumosen. Stud. from the labor. of phys. Ch. Yale university. II. New Haven 1887. Bd. 24.

4) Contejean, Pepsinverdauung d. Albùmins. Arch. de physiol.

5) Neumeister, Globulin und Globulosen, Z. f. Biol. Bd. 22. $18 \varepsilon 6$.

6) Kühne u. Chittenden, Myosin und Myosinosen. Zeitschrift für Biol. Bd. 25. 1889.

7) v. Fürth, Ueber die Eiweisskörper des Muskelplasmas. Arch. f. exp. Path. u. Pharm., Bd. 36. 1895.

8) Chittenden u. Hart, Elastin und Elastosen. Zeitschr. f. Biol. Bd. 25. 1889. 
So werthvoll auch die von den genannten Autoren an derartigen Gemengen erhaltenen Resultate sind, so bestand doch die Verpflichtung, die homogenen Eiweissstoffe, die uns heute in reinerer Form zur Verfügung stehen, zur Untersuchung heranzuziehen: vor Allem das krystallinisch dargestellte Eieralbumin und Serumalbumin; ihnen habe ich noch ein möglichst reines, wenn auch nicht krystallinisches Serumglobulin zugesellt. Da die nähere chemische Untersuchung der von Pick dargestellten Verdauungsprodukte noch im Gang ist, habe ich von einer Reindarstellung und Analyse der aus den verwendeten Proteinstoffen gewonnenen Spaltprodukte zunächst Abstand genommen und nur Antwort auf die Eingangs gestellte Frage nach Zahl und Qualität der peptischen Verdauungsprodukte gesucht.

\section{Eieralbumin.}

Chittenden und Bolton ${ }^{1}$ ) haben aus ihrem Eieralbumin durch Pepsinspaltung Produkte erhalten, die sich, nach ihrer Aussage, nicht wesentlich unterscheiden von den analogen Fibrinprodukten Kühne's. Sie wurden gemäss ihren Gattungseigenthümlichkeiten, auf Grund deren ihre Darstellung erfolgte, als primäre Albumosen bezeichnet, soweit sie durch Kochsalzsättigung der vom Neutralisationspräcipitat befreiten Verdauungslösung gewonnen waren, als Deuteroalbumosen, soweit sie aus dem salzgesättigten Filtrat durch salzhaltige Essigsäure abgeschieden werden konnten, der Rest als Peptone.

Ihre primäre Albumose erwies sich als ein Gemenge von unlöslicher Dysalbumose, von Heteroalbumose, die sich erst bei der Dialyse ausschied, und von Protoalbumose, die auch in salzfreiem Wasser löslich blieb.

Die Pepsinspaltung, die Contejean ${ }^{2}$ ) an seinem coagulirten Eiereiweiss vornahm, lieferte ihm zunächst beim Neutralisiren der Verdauungsflüssigkeit Syntonin, durch Kochsalzsättigung in salpetersaurer Lösung Deuteralbumosen und endlich

1) l. c. 
Pepton. Das Auftreten von Protoalbumose erschien ihm unsicher, Heteroalbumose fand er nicht.

Was den Gang vorliegender Untersuchungen anbelangt, so wurde zunächst behufs Darstellung des krystallinischen Eiereiweisses nach den letzten darauf bezüglichen Angaben $\mathrm{Hof}-$ meisters ${ }^{1}$ ) verfahren. Es mag hier noch einmal betont sein, dass dieselbe erheblich beschleunigt wird, wenn man die wässerigen Lösungen der umzukrystallisirenden Niederschläge nach dem Versetzen mit der nothwendigen Menge gesättigter Ammonsulfatlösung mit einigen Krystallen von Eiereiweiss impft. Das Ausfallen der Krystalle geht dann vollkommen und in wenigen Stunden vor sich. Es wurde so oft umkrystallisirt; bis sich der schneeweisse Niederschlag aus reinen, gleichmässigen Krystallformen, frei von amorphen Beimengungen, zusammensetzte. Die Ausbeute von 35 Eiern betrug, scharf abgepresst gewogen, 24,2 gr., wovon $55 \%$ reines, wasserfreies Eiweiss waren.

Die Krystallmasse wurde in Wasser gelöst, so dass die Lösung $4 \%$ Eiweiss enthielt, diese Lösung auf einen Salzsäuregehalt von $0,5 \%$ gebracht und mit etwas Pepsinum purissimum(Grübler) versetzt. Verunreinigungen der Verdauungsprodukte durch dieses Präparat sind ausgeschlossen, denn eine $1 \%$ ige Lösung desselben bleibt auf Zusatz von verdünntem Kupfersulfat oder Salpetersäure und Kochsalz vollkommen klar, Sättigung mit Ammonsulfat bewirkt nur ganz schwache Opalescenz. Die Biuretreaction ist ganz schwach positiv.

Die Verdauungslösung wurde dann in gleichmässiger Temperatur von $38-40^{\circ} \mathrm{C}$. gehalten, so lange, dass sich einerseits noch Protoalbumosen, andererseits schon Peptone darin nachweisen lassen. Damit letztere einigermassen reichlich vorhanden sind, bedarf es beim Eieralbumin immerhin einer 4-6tägigen Verdauung, je nach der Menge des zugesetzten Pepsins.

Auffallender Weise entsteht, wenn man eine ammon-

1) Hofmeister, Ueber jodirtes Eieralbumin. Zeitschr. f. phys. Chemie. Bd. XXIV. 1897. 
sulfathaltige Lösung benützt, in der Anfangs klaren Verdauungsflüssigkeit sehr bald eine gleichmässige, milchige Trübung, die schnell zunimmt, bis sich das Ganze in eine opalescente, kleisterartige Masse verwandelt hat, die beim Ausgiessen in voluminösen Klumpen an den Gefässwandungen haftet. Dieser Eiweisskörper hat, isolirt und in gereinigtem Zustand, die Eigenschaften eines Acidalbumins mit besonders intensiver Furfurolreaction und tritt so augenfällig bei der Verdauung hervor, weil er sich in saurer Flüssigkeit schon bei geringem Gehalt derselben an Ammonsulfat vollkommen ausscheidet. Er wird von der Verdauung weiterhin nur sehr schwer angegriffen und mit Pepsinsalzsäure im Brutschrank erst nach mehreren Tagen merklich zu Albumosen und Peptonen abgebaut. Dialysirt man jedoch die Lösung der Eiweisskrystalle, ehe man an die Digestion geht, bis zur möglichsten Entfernung des Ammonsulfats, so tritt im Lauf der Verdauung nur eine ganz schwache Trübung auf und die kleisterartige Abscheidung bleibt aus, dagegen schreitet die Peptonisirung viel rascher fort, so dass man die bereits bekannte, langsame Verdauung salzhaltiger Eiweisslösungen wohl auf die Bildung solcher schwer angreifbaren Abscheidungen von «Acidalbuminen» beziehen muss. Es scheint, als ob dieselben in einer gewissen Beziehung zur Heteroalbumose, d. h. dem wasserunlöslichen Theil der primären. Albumosen ständen: in der ammonsulfathaltigen Verdauungslösung war nach Entfernung dieser kleisterartigen Syntoninausscheidung Heteroalbumose im Filtrat nicht mehr vorhanden, während sie im Filtrat der salzfreien Verdauungslösung reichlich nachzuweisen war.

Die durch Filtriren von dem ausgeschiedenen Acidalbumin befreite, klare Verdauungslösung ergibt beim Neutralisiren mit Ammoniumcarbonat kein Neutralisationspräcipitat mehr und bleibt heim Aufkochen klar. Erst beim Einengen im Wasserbad auf halbes Volumen scheiden sich noch geringe Reste von Acidalbumin in spärlichen feinen Flöckchen aus.

Die Trennung und Isolirung der Albumosen und Peptone geschah durchweg nach dem von E. P. Pick ${ }^{1}$ ) ausgearbeiteten

1) $1 . \mathrm{c}$. 
Verfahren; es beruht auf fractionirter Fällung derselben mit Ammonsulfatlösung und zwar ist die vollkommene Aussalzung einer Albumosenfraction an einen constanten Concentrationsgrad des Sulfats in der Gesammtlösung, die sogenannte obere Fällungsgrenze, gebunden. Es wurde dabei gleichzeitig geprüft, inwieweit sich dieselbe bei den verschiedenen Eiweissarten verschiebt.

Betreffs Handhabung der Methode sei auf die angeführte Arbeit verwiesen.

Als untere und obere Fällungsgrenzen für die primären Albumosen, Fraction I, in neutraler Lösung ergaben sich 3,6 und 4,6, d. h. also die Fraction I der Eieralbumosen ist in. einer ammonsulfathaltigen Flüssigkeit vollkommen ausgesalzen, wenn darin das Verhältniss von gesättigter Sulfatlösung zu wässeriger Eiweisslösung - deren Concentration in weiten Grenzen schwanken darf - auf 4,6:5,4 eingestellt wird. Da bei einem Verhältniss von $5: 5$ die nächste Fraction noch vollkommen in Lösung bleibt, weil die untere Grenze ihrer Fällung höher liegt, so versetzt man also die vollkommen klare, genau neutrale Verdauungslösung mit dem gleichen Volumen gesättigter Ammonsulfatlösung, wobei dann die primäre Albumose flockig ausgeschieden wird. Nach mehrstündigem Stehen setzt sie sich ziemlich reichlich am Boden des Gefässes ab und kann leicht von der überstehenden klaren Flüssigkeit abfiltrirt werden. Der Niederschlag löst sich bis auf einige unlösliche opake Schollen, Spuren von Heteroalbumose, in destillirtem Wasser und lässt sich so durch mehrfaches Lösen und Fällen reinigen. Man erhält schliesslich einen schneeweissen Niederschlag, der, abgepresst und dann in Wasser gelöst, zur Anstellung der zur Charakterisirung der Fraction nöthigen Reactionen dienen kann. Nach mehrtägigem Dialysiren der Fraction in fliessendem Wasser erfolgte keine Abscheidung von Heteroalbumose mehr, während, wie bereits oben erwähnt, in der durch Dialyse ammonsulfatfrei gemachten Verdauungslösung ein grösserer Theil der Fraction I wasserunlösliche Heteroalbumose war. Diese Heteroalbumose fällt durch eine unverhältnissmässig starke, auf 
die Anwesenheit von Kohlehydratgruppen zu beziehende Violettfärbung beim Versetzen mit $\alpha$-Naphtol und concentrirterSchwefelsäure auf und erinnert hierdurch abermals an ihre erwähnte Beziehung zum beschriebenen Acidalbumin.

Der wasserlösliche Theil der Fraction I zeigt alle Eigenschaften der Kühne'schen Protoalbumosen. Er wird mit verdünntem Kupfersulfat gefällt, ebenso mit Essigsäure und Ferrocyankalium, nur unvollkommen mit Kochsalz in neutraler Lösung und unvollkommen mit Salpetersäure. Salzsättigung in saurer Lösung fällt vollkommen. Alle diese Fällungen sind in der Hitze löslich und kehren beim Erkalten wieder. Die den Albumosen als Proteinstoffen zukommenden Eigenschaften, so ihr Verhalten gegenüber den Alkaloidreagentien, das starke Hervortreten der Millon'schen und Biuretreaction, sind unverkennbar. Dagegen fällt Molis ch's, sowie die Schwefelreaction nur ganz schwach aus.

Die untere resp. obere Fällungsgrenze der. Fraction II liegt bei 5,6 resp. 6,0, so dass sie vollkommen ausgesalzen ist, wenn die Gesammtlösung zu $2 / 3$ Volumen mit Ammonsulfat gesättigt ist. Sie ist etwa in gleicher Menge wie Fraction I vorhanden und setzt sich nach mehrstündigem Stehen als feiner, sandiger, schneeweisser Niederschlag am Boden ab. Die durch mehrfaches Lösen und Fällen gereinigte Albumose besteht aus lauter grösseren, kleineren und kleinsten Globulitenformen, die jedoch weder strahliges Gefüge noch Doppelbrechung erkennen lassen. Krystallisationsversuche waren erfolglos. Sie hat die Eigenschaften der Deuteroalbumosen im Kühne'schen Sinne. Sie wird durch Sättigung mit Kochsalz in salpetersaurer Lösung vollkommen ausgeschieden, dagegen von Salpetersäure oder Kochsalz allein überhaupt nicht, ebensowenig durch verdünntes Kupfersulfat, unvollkommen durch Essigsäure und Ferrocyankalium. Die Schwefel- ebenso wie die Molisch'sche Reaction tritt im Gegensatz zu der starken Millon'sche Reaction wenig hervor.

Weitaus am reichlichsten ist die Fraction III vorhanden, die durch Sättigen mit pulverförmigem Ammonsulfat in neutraler Lösung gewonnen wird. Ihre Fällungsgrenzen sind 7,0 und 7,8. Nach einiger Zeit scheidet sie sich als zusammenhängende weisse 
Kruste ab, die auf klarer Flüssigkeit schwimmt. Die gereinigte Albumose ist amorph und enthält einzelne Globuliten. Bei allmählichem, wochenlangem Eindunsten einer wässerigen Lösung, die mit Ammonsulfat etwa halb gesättigt worden war, kann man erhebliche Zunahme der Globuliten beobachten, sie wachsen zu grösseren, stark lichtbrechenden Kugeln an, die jedoch von Doppelbrechung oder strahliger Säumung des Randes, wie man sie in den Vorstadien von Krystallisationen zu sehen bekommt, nichts erkennen lassen. Diese Fraction verhält sich in Bezug auf ihre Eigenschaften wie eine Deuteroalbumose, zeichnet sich aber durch eine ausserordentlich intensive Molisch'sche Reaction vor allen andern Fractionen aus: beim Versetzen mit 1 Tropfen . $\alpha$-Naphtol und concentrirter Schwefelsäure tritt zunächst ein tief rothvioletter Ring an der Berührungsstelle von Säure und Flüssigkeit auf, und beim Umschütteln färbt sich die ganze Flüssigkeit tief violett.

Das salzgesättigte Filtrat enthält eine IV. Fraction und zwar wurde die Beobachtung gemacht, dass dieselbe daraus nicht allein durch salzgesättigte Schwefelsäure abgeschieden werden kạnn, wie für die entsprechende Fraction aus sogenanntem Witte-Pepton von E. P. Pick festgestellt worden ist, sondern ebensowohl durch einen bestimmten Zusatz salzgesättigten Ammoniaks. Zu diesem Zweck wurde eine schwache Ammoniaklösung mit Ammonsulfat gesättigt und ihre Alkalescen $z$ bestimmt: $1 \mathrm{ccm} .=0,58$ Normalschwefelsäure $=2,7 \mathrm{ccm}$. der ammonsulfatgesättigten verdünnten Schwefelsäure (die zur Abscheidung der Albumose in saurer Lösung benutzt wurde). $2 \mathrm{ccm}$. des salzgesättigten neutralen Filtrats wurden nun mit dieser Ammoniaklösung versetzt und zwar in zunehmenden Mengen von 1 Tropfen an beginnend. Es ergab sich in der autgestellten Reihe eine Trübung, die bei $0,1 \mathrm{ccm}$. Ammoniaklösung in $2 \mathrm{ccm}$. Albumoselösung begann und bei 0,4 in $2 \mathrm{ccm}$. am intensivsten war und sich flockig absetzte. So lange die Ausscheidung in der aufgestellten Reihe noch unvollkommen war, konnten in den Filtraten die Reste der Albumose sowohl durch salzgesättigtes Ammoniak wie durch salzgegesättigte Schwefelsäure völlig abgeschieden werden. Erst in 
dem Filtrat derjenigen Probe, in welchem das Optimum des Ammoniakzusatzes erreicht schien, war weder durch Säure noch durch Ammoniak eine weitere Abscheidung zu erzielen. Im Ueberschuss der salzgesättigten Ammoniaklösung löst sich die Fällung wieder. Versetzt man umgekehrt, den Angaben von E. P. Pick gemäss, das neutrale salzgesättigte Verdauungsfiltrat mit salzgesättigter Schwefelsäure in allmählich steigender Menge, so erreicht man vollständige Abscheidung der Albumose bei Zusatz von $1 /{ }_{10}$ Volumen salzgesättigter Säure zu dem Gesammtvolumen der Verdauungslösung. Im Ueberschuss von Säure tritt allmähliche Lösung ein. Reste der Albumose in Filtraten von Proben mit ungenügendem Säurezusatz können ebensowohl durch salzgesättigte Säure wie Ammoniak vollends ausgeschieden werden.

Man sieht also, die Fraction wird aus dem neutralen salzgesättigten Verdauungsfiltrat gewonnen, wenn die Reaction sich ändert, sei es, dass sie sauer, sei es, dass sie alkalisch wird. Es bedarf jedoch eines bestimmten Alkalescenz- oder Säuregrades, damit eine vollständige Abscheidung erfolgt, wird dieser Grad nach unten oder oben hin überschritten, so bleiben Albumosenreste in Lösung. Beim Eieralbumin bedarf es dabei eines verhältnissmässig höheren Alkalescenz- als Säuregrades. Diese Erfahrung erklärt eine Angabe Kühne's'), welcher im salzgesättigten Filtrat anscheinend zwei Albumosen isolirte, die eine dadurch, dass er das Filtrat mit Ammoniak und Ammoniumcarbonat "kräftig alkalisch" machte und in der Hitze mit Ammonsulfat sättigte, die andere, indem er das daraus erhaltene Filtrat mit salzgesättigter Essigsäure versetzte unter Vermeidung eines Ueberschusses. Die Albumose war wohl nicht völlig, in Folge des unbestimmten Alkalescenzgrades, abgeschieden und ihr Rest dann durch die Säure gewonnen worden.

Dieses Verhalten der Fraction IV weist auf einen amphoteren, also sowohl säure- wie alkalibindenden Charakter hin, wie er den Amidosäuren eigenthümlich ist.

1) Erfahrungen über Albumosen und Peptone von W. Kühne. Zeitschr. f. Biol. Bd. XXIX, 1892. 
Die aus dem neutralen Verdauungsfiltrat durch $1 / 10$ Volumen salzgesättigte Säure abgeschiedene Albumose ist niemals sehr reichlich. Sie tritt gewöhnlich als trübe Opalescenz auf, um sich nach einigen Stunden in feinen Flöckchen abzusetzen, die sich leicht auf dem Filter sammeln lassen.

Ihre wässerige Lösung bleibt auf Zusatz von verdünntem Kupfersulfat, von Salpetersäure, von Kochsalz vollkommen klar. Sättigt man mit Kochsalz und säuert man mit Salpetersäure an, so entsteht eine kaum erkennbare, äusserst feine Trübung, die wohl auf geringe Reste der Fraction III zurückzuführen ist, ebenso wie die minimalen 'Spuren von nichtoxydirtem Schwefel, die beim Kochen mit Lauge und Blei eine ganz. schwache Bräunung verursachen. Die Anwesenheit der Oxyphenylgruppe wird beim Kochen mit Millon's Reagens durch die intensiv rothgelbe Färbung deutlich. Die Kohlehydratreactionen treten besonders stark hervor, so dass beim Versetzen mit $a$-Naphtol und concentrirter Schwefelsäure ein intensiver dunkelvioletter Ring, mit Eisessig und concentrirter Schwefelsäure ein schönes Rothblau entsteht. Diese Fraction nimmt eine Zwischenstellung zwischen den Albumosen und Peptonen ein.

Das albumosenfreie, saure Filtrat enthält nun noch, wie die starke Biuretreaction anzeigt, Peptone, und zwar ist auch hier wieder durch Fällung mit Jod ein Gemenge von 2 Peptonen abzuscheiden, von denen das eine in Alkohol unlöslich, das andere in Alkohol löslich ist. Die Isolirung geschah wie bei Pick.

Fraction V, der in Alkohol unlösliche Theil des Jodjodkaliumniederschlages ist stets ziemlich reichlich und leicht durch wiederholtes Waschen und Fällen mit Alkohol und Ausschütteln mit Aether vollkommen jod- und auch jodkaliumfrei zu erhalten, so dass durch Zusatz von salpetriger Säure kein Jod mehr nachzuweisen ist. Die Fraction hat eine zartröthliche, lachsartige Farbe und fluorescirt in wässeriger Lösung grünlich. Durch verdünntes Kupfersulfat, durch Salpetersäure, durch Salpetersäure und Kochsalz, durch Essigsäure und Ferrocyankalium ist sie nicht mehr fällbar, wohl aber durch die Alkaloidreagentien wie Jodquecksilberkalium, Trichloressigsäure, Gerb- 
säure. Die Fällungen sind in der Hitze und im Ueberschuss des Fällungsmittels mehr oder weniger löslich und kehren beim Erkalten wieder. Gerbsäure löst im Ueberschuss nicht. Die Fraction bräunt sich beim Kochen mit Blei und Lauge nicht mehr. Mit Millon's Reagens ist nur noch eine schwache Rothgelbfärbung wahrnehmbar, um so auffallender tritt eine höchst intensive Molis ch'sche Reaction hervor. Die Adamkiew ic z'sche Reaction fällt negativ aus, vermuthlich weil die eine der beiden Vorbedingungen $\mathrm{zu}$ ihrem Zustandekommen, die aromatische Gruppe, hier ganz zurücktritt. ${ }^{1}$ ) Xanthoprotein- und Biuretreactionen sind intensiv.

Der alkohollösliche Theil des Jodjodkaliumniederschlags, Fraction VI, ist seiner Quantität nach nicht sehr erheblich. Durch Ausschütteln mit Aether lässt er sich nicht völlig jodfrei machen, kocht man jedoch die wässerige neutrale Lösung mit Bleioxyd auf, so ist nach dem Erkalten in dem wasserhellen bleifreien Filtrat, auch mit salpetriger Säure, Jod nicht mehr nachzuweisen. Es muss darauf geachtet werden, da z. B. die Furfurolreactionen, die Millon'sche Reaction u. A. an jodhaltigen Präparaten nicht ausführhar sind.

Diese Fraction unterscheidet sich von der vorhergehenden vor Allem dadurch, dass eine äusserst schwache Molisch'sche Reaction höchstens nur auf Spuren einer Kohlehydratgruppe hinweist. Vom aromatischen Atomcomplex sipd durch Millon's Reagens noch Spuren nachzuweisen. Im Uebrigen ist sie gleichfalls anscheinend frei von nichtoxydirtem Schwefel, verhält sich zu. den Alkaloidreagentien ebenso und gibt intensive Xanthoprotein- und Biuretreaction.

Die vorliegenden Resultate sind an mehreren Portionen krystallinischen Eiweisses, die gesondert verdaut wurden, gewonnen. Ausserdem wurde noch ein "Eieralbuminpepton" von König u. Comp., das als Gemenge von Verdauungsprodukten aus Eieralbuminen und Globulin zu betrachten ist, in gleicher Weise untersucht. Es ergab sich, dass sich dasselbe ebenfalls aus den beschriebenen 6 Fractionen zusammensetzt, und zwar sind die Fällungsgrenzen der einzelnen genau dieselben wie

1) cf. Ueber jodirtes Eieralbumin von F. Hofmeister. Z. f. phys. Ch. Bd. XXIV. 1897. 
bei denjenigen des krystallinischen Albumins, Die einzelnen Albumosen zeigen jedoch nicht die schöne schneeweisse Farbe, sie haben einen gelbbraunen Ton und sind schwerer auf dem Filter zu sammeln. In Bezug auf ihre sonstigen Eigenschaften gleichen sie denjenigen des krystallinischen Albumins, nur sei besonders erwähnt, dass die Heteroalbumose, die in mässiger Menge daraus isolirt wurde, sich durch sehr intensive Molisch'sche Reaction auszeichnete, dass ferner die Säurefraction (IV) frei von abspaltbarem Schwefel erschien.

\section{Serumalbumin.}

Es wurde dasselbe nach den Vorschriften von Gürber ${ }^{1}$ ) ebenfalls krystallinisch dargestellt und durch Umkrystallisiren gereinigt, bis der Niederschlag sich unter dem Mikroskop voll-: kommen frei von amorphen Beimengungen erwies. Im Ganzen ist er schwach gelblich gefärbt in Folge von sehr hartnäckig anhaftenden Spuren eines Gallenfarbstoffs, der allmählich in blassgrün übergeht. Die einzelnen durch die Verdauung gewonnenen Fractionen sind deshalb auch nicht von so schneeweissem Aussehen wie beim Eieralbumin, sondern haben einen mehr gelblich grauen Farbenton. Die wässerige gelbe, etwas ins Grünliche opalisirende Lösung der Serumkrystalle, die 3\% Eiweiss enthielt, wurde zunächst in fliessendem Wasser analysirt, dann auf einen Salzsäuregehalt von $0,5^{n} / 0$ gebracht und unter Zusatz von Pepsinum purissimum (Grübler) - auf 1/2 l. Flüssigkeit $0,5 \mathrm{gr}$. - bei constanter Temperatur von $38-40^{\circ}$ G. verdaut.

Im Gegensatz zum Eieralbumin geht hier die Verdauung bedeutend schneller vor sich, und die Abscheidung des schwerlöslichen Acidalbumins, wie sie beim Eieralbumin als weisse Gallerte erfolgte, ist selbst in nicht dialysirter, also sulfathaltiger Verdauungsflüssigkeit, nur ganz unerheblich. Die Bildung dieses für die weitere Verdauung so schwer angreifbaren Acidalbumins,

1) Gürber, Krystallisation des Serumalbumins. Sitzungsber. d. phys.-med. Ges. z. Würzburg. 1894. In dem 1897 erschienenen «Leitfaden der Physiologie von Schenck und Gürber ist ganz kurz bemerkt (S. 23), dass das Serumalbumin in wenigstens 7 schwefelhaltige Verdauungsprodukte zerfällt. Der näheren Mittheilung Gürber's darf mit besonderem Interesse entgegengesehen werden. 
dessen Beziehung zur Heteroalbumose wir schon gedachten, scheint bei den verschiedenen Eiweissarten für ihre «Schwer-» oder "Leichtverdaulichkeit» entschieden bedeutsam zu sein. Es wurden 4 verschiedene Verdauungsversuche mit krystallinischem Albumin aus dem Serum verschiedener Pferde vorgenommen und in keinem Fall nur annähernd die Abscheidung der Syntoningallerte wie beim Eieralbumin beobachtet; eine schwache Opalescenz, nicht mehr. Beim Neutralisiren nimmt dieselbe etwas zu und setzt sich beim Kochen feinflockig ab. Aber man gewinnt dabei stets, in welchem Stadium man auch die Verdauung unterbrechen mag, nur geringe Mengen. Es muss also dieser Eiweisskörper bei der Verdauung des Serumalbumins sehr schnell weiter gespalten werden, fast im Moment der Entstehung, oder aber - was wahrscheinlicher - er stellt nur für einen Theil der Spaltprodukte, nämlich für die resistentere Heteroalbumose, eine Vorstufe dar, während die grössere Menge derselben dieses intermediäre Stadium nicht durchläuft. Die gereinigte Substanz hat durchaus die Eigenschaften eines Acidalbumins und zeichnet sich wiederum durch eine sehr starke Reaction nach Molisch aus.

Das neutrale, klare Verdauungsfiltrat enthält zunächst eine Fraction I, deren untere Fällungsgrenze bei 4,2 , die obere bei 4,6 liegt. Es bedarf also eines verhältnissmässig hohen Procentsatzes an Ammonsulfat, damit ihre Aussalzung beginnt, während die obere Grenze der Fraction mit den bisher bekannten übereinstimmt. Diese Albumose ist jedoch stets beim Serumalbumin nur in so geringen Mengen vorhanden, dass sie sich beim Versetzen der Verdauungslösung mit gleichem Volumen Sulfat nur als schwache Opalescenz abscheidet, gleichgültig, ob man sie nach 3 oder 18 stündiger Verdauung zu fassen sucht. Es hat somit den Anschein, als ob sie keineswegs für alle folgenden, sehr reichlich vorhandenen Albumosen ein Durchgangsstadium darstellt, es müsste denn sein, dass sie im Entstehen schon wieder weitergespalten wird.

Die Fraction wurde durch wiederholtes Lösen und Fällen gereinigt und an ihren Eigenschatten als primäre Albumose erkannt. Sie fällt mit verdünntem Kupfersulfat, mit Kochsalz, 
mit Salpetersäure, wenn auch unvollkommen, aus ihrer wässerigen Lösung. Bemerkenswerth ist der ausserordentlich reichliche Niederschlag von Schwefelblei beim Kochen mit Bleiacetat und Natronlauge. Mit Schwefelsäure und $\boldsymbol{- N}^{-N a p h t o l}$ tritt nur sehr spärliche Rothfärbung auf. Die klare wässerige Lösung trübt sich in der Dialyse, aber eine Abscheidung von Heteroalbumose an den Wandungen des Pergamentschlauches ist nicht zu erzielen. Sie mag immerhin - nach dem spärlichen Auftreten des Acidalbumins zu schliessen - in geringen Mengen vorhanden sein.

Die Fraction II mit der unteren Fällungsgrenze 5,4, der oberen 6,2 tritt beim Versetzen des klaren Filtrats der vorhergehenden Fraction mit 1/2 Volumen gesättigter Ammonsulfatlösung als intensive milchige Trübung von schleimiger Beschaffenheit auf. Die Trübung bleibt selbst nach mehrtägigem Stehen ganz diffus und ist sehr mühsam abzufiltriren, da sie sich aus kleinsten Tröpfchen zusammensetzt, die die Poren des Papiers leicht passiren. Die wässerige Lösung der durch wiederholtes Lösen und Fällen gereinigten Fraction bleibt auf Zusatz von verdünntem Kupfersulfat, wie von Salpetersäure klar, durch Essigsäure und Ferrocyankalium entsteht schwache Trübung, durch Kochsalzsättigung eigenthümlicher Weise eine flockige Fällung, als ob diese Fraction eine Mittelstellung zwischen primären und Deuteroalbumosen einnähme. Beim Kochen mit Bleiacetat und Kalilauge fallen so grosse Mengen Schwefelblei, dass die gạnze Flüssigkeit dick schwarz wird, während wiederum - wie bei Fraction I - die Molisch'sche Reaction nur sehr schwach ausfällt.

Bei 7,2 beginnt Fraction III zu fallen und ist bei 8,0 vollkommen ausgesalzen. Sie wird gewonnen aus dem Filtrat der Fraction II durch Sättigung mit gepulvertem Ammonsulfat, scheidet sich dabei sehr reichlich in dicken Flocken ab, die nach einigem Stehen $\mathrm{zu}$ grauweisser, auf der Oberfläche schwimmender Kruste zusammenbacken. Die wässerige Lösung trübt sich mit verdünntem Kupfersulfat nicht mehr, ebenso wenig wie mit Salpetersäure oder Kochsalz, deutlich mit Essigsäure und Ferrocyankalium, dick flockig mit Kochsalz in salpetersaurer Lösung, zeigt also die Eigenschaften einer Deuteroalbu- 
mose. Die Mengen abspaltbaren Schwefels, die als Schwefelblei aus alkalischer Bleilösung ausgeschieden werden, sind geradezu enorm, während auch hier wieder die Furfurolreactionen nur sehr schwach ausfallen. Von den Alkaloidfällungsmitteln fällt Trichloressigsäure nur noch sehr unvollkommen, vollständig jedoch Jodquecksilberkalium und Gerbsäure.

Ebenso wie beim Eieralbumin enthält auch hier das salzgesättigte Filtrat eine Fraction IV von amphoterem Charakter, die sich abscheiden lässt, indem man entweder mit $1 / 10 \mathrm{Vol}$. salzgesättigter Säure oder mit 1/10 Vol. salzgesättigten Ammoniaks, der erwähnten Concentration, versetzt. Die in verhältnissmässig geringen Mengen vorhandene, gereinigte Fraction steht den Albumosen in ihren Eigenschaften näher, als es beim Eieralbumin der Fall ist, insofern sie auch durch Kochsalzsättigung in saurer Lösung, desgleichen mit Essigsäure und Ferrocyankalium - wenn auch unvollkommen - gefällt wird. Beim Kochen mit Blei in alkalischer Lösung fällt massenhaft schwarzes Schwefelblei, beim Kochen mit Millon's Reagens bilden sich schöne himbeerrothe Flocken, dagegen ist die Violettfärbung auf Zusatz von $\alpha$-Naphtol und concentrirter Schwefelsäure kaum noch erkennbar. Jodquecksilberkalium, sowie Gerbsäure fällen vollkommen, ersteres löst im Ueberschuss völlig, letzteres zum Theil. Die Fällungen lösen sich in der Wärme, um beim Erkalten wiederzukehren. Trichloressigsäure fällt nicht mehr.

In dem albumosenfreien Filtrat sind auch hier wieder und zwar in sehr reichlichen Mengen die beiden durch Jod fällbaren und durch ihr Verhalten zum absoluten Alkohol leicht zu trennenden Peptone enthalten.

Fraction V, das alkoholunlösliche, ist durch Waschen und Fällen mit Alkohol und Schütteln mit Aether leicht jodfrei darzustellen und zeichnet sich wiederum durch eine schöne rosarothe Lachsfarbe aus. Diese Fraction unterscheidet sich von der vorhergehenden wesentlich, indem abspaltbarer Schwefel nur noch in verschwindenden Mengen nachweisbar ist, dagegen die Rothviolettfärbung beim Anstellen der Molisch'schen Reaction augenfällig zu Tage tritt. Fällbar ist sie - abgesehen 
von Jod und Alkohol - nur noch durch die Alkaloidreagentien, ausgenommen Trichloressigsäure.

In der alkohollöslichen Fraction VI ist unoxydirter Schwefel überhaupt nicht mehr nachweisbar und nur geringe Violettfärbung mit $\boldsymbol{\alpha}$-Naphtol und Schwefelsäure. Die Oxyphenylgruppe verursacht noch schöne Rothfärbung der Millon'schen Lösung. Auch den Alkaloidreagentien, Jodquecksilberkalium und Trichloressigsäure, gegenüber verhält sie sich indifferent und fällt nur noch unvollkommen mit Gerbsäure. Beim Kochen mit Salpetersäure entsteht eine intensive Gelbfärbung, die bei Zusatz von Natronlauge vollkommen bis zur Farblosigkeit verschwindet.

\section{Serumglobulin.}

Kühne und Chittenden haben bei der peptischen Spaltung ihres Globulins, das freilich aus den Eingangs erwähnten Gründen nicht als einwandfreies Ausgangsmaterial gelten kann, ähnliche Resultate wie bei der Fibrinverdauung gewonnen. Sie erhielten aus der neutralen Verdauungsflüssigkeit, nach Entfernung unverdauter Globulinreste und Siedecoagulate aus verdautem Globulin, eine durch Kochsalz und Steinsalzsättigung fällbare "Protoglobulose», die sich von der entsprechenden Fibrinalbumose dadurch unterschied, dass ihre neutrale wässerige Lösung, mit wenig Kochsalz zum Sieden erhitzt, eine starke Trübung ergab, welche sich beim Abkühlen klärte, ein Verhalten, das sie als umgekehrte Albumosenreaction bezeichneten. Beim Dialysiren der Salzfällung aus der neutralen Verdauungsflüssigkeit schied sich eine Heteroglobulose aus, die bei weiterer tryptischer Verdauung reichlich Pepton und Spuren von Leucin gab, während die Protoalbumose tryptisch in Leucin und Tyrosin gespalten werden konnte. Aus dem salzgesättigten Filtrat wurde durch Essigsäure eine Deuteroglobulose abgeschieden, die der Fibrindeuteroalbumose analog war. Das Präparat, von dem Kühne und Chittenden ausgingen, erwies sich übrigens gegen die Pepsinverdauung als ausserordentlich resistent.

Kühne und seine Schüler haben die Albumosen verschiedener Provenienz durch specielle Benennungen unterschieden als Globulosen, 
Myosinosen, Elastosen, Vitellosen. In Anbetracht der Erfahrung, die sich auch aus vorliegender Arbeit entnehmen lässt, dass die primären und secundären Spaltungsprodukte verschiedener Herkunft mehr oder weniger analog, möglicher Weise zum Theil identisch sind, halten wir es für zweckmässig, vorläufig nur von Albumosen, primären und secundären, zu sprechen. Auch der von Chittenden in die Nomenclatur eingeführte, aber wenig eingebürgerte Ausdruck Proteosen würde denselben Zweck erfüllen.

Wie Untersuchungen von Kauder ${ }^{1}$ ) und Reye ${ }^{2}$ ) gezeigt haben, gelingt es, die 3 Eiweisskörper des Blutplasmas, das Fibrinogen, Globulin und Serumalbumin, durch fractionirte Fällung mit Ammonsulfat vollkommen von einander zu trennen. Pferdeblutplasma, das durch Auffangen des frischen Blutes in Ammoniumoxalatlösung am Gerinnen verhindert ist, wird nach Absetzen der morphologischen Elemente decantirt und mit 1/3 Volumen gesättigter Ammonsulfatlösung versetzt, dann hat man sicher auch fibrinogenähnliche Substanzen ausgesalzen, freilich schon - wenn man mit unverdünntem Plasma arbeitet auch einen geringen Theil des Globulins. Führt man weiter Ammonsulfat in das Filtrat ein bis zur halben Sättigung desselben, so fällt das Globulin in dicken Flocken, während das Serumälbumin in Lösung bleibt. Der Niederschlag wird auf dem Filter gesammelt, mit halbgesättigter Ammonsulfatlösung gewaschen und dreimal aus der wässerigen Lösung umgefällt. Die letzte Fällung löst sich leicht in destillirtem Wasser, die Lösung erscheint im durchfallenden Licht gelb, im auffallenden stark grünlich opalisirend. Mit dieser klaren Globulinlösung wurde ein Krystallisationsversuch vorgenommen, derart, dass sie bis zur beginnenden Trübung mit Ammonsulfat versetzt und dann einer langsamen Verdunstung überlassen wurde. Im Laufe von 8 Tagen hatte sich das Globulin in schönen, auffallend grossen Globuliten ausgeschieden, die jedoch nicht doppelbrechend oder strahlig gefügt waren und auch weiterhin keine Neigung zu echter Krystallisation zeigten.

Bringt man die wässerige Globulinlösung auf einen Salz-

1) Kauder, Zur Kenntniss der Eiweisskörper des Blutserums. Archiv f. exp. Path. u. Pharm., Bd. $\dot{2} \dot{6}$.

2) Reye 1. c. 
säuregehalt von $0,5 \%$, so tritt fast augenblicklich eine milchige Trübung ein und die ganze Flüssigkeit verwandelt sich nachher unter der Digestion mit Pepsin in einen opalescenten Kleister, der sich nicht filtriren lässt. Allmählich setzt sich derselbe, nach mehrtägiger Digestion, in weissen Flocken unter der klar überstehenden Verdauungsflüssigkeit $a b$ und kann nun leicht auf dem Filter gewonnen werden. Er stellt ein Acidglobulin dar von schwach sauren Eigenschaften und löst sich in Wasser nach Neutralisiren. Schon bei gering alkalischer Reaction ist er schnell löslich und wird durch Ansäuern mit verdünnten Säuren leicht als schöner, weisser flockiger Niederschlag wieder ausgefällt. Der so gereinigte Körper verhält sich in Bezug auf seine Löslichkeit ganz ebenso und fällt in schwach saurer Lösung bei Anwesenheit geringster Mengen Ammonsulfat völlig aus. $0,1 \mathrm{ccm}$. einer gesättigten Ammonsulfatlösung genügen schon, um aus $5 \mathrm{ccm}$. schwach saurer Flüssigkeit das Acidglobulin vollständig auszusalzen. Daher erklärt sich auch hier - ähnlich wie beim Eieralbumin seine Abscheidung in der ammonsulfathaltigen sauren Verdauungslösung während der Digestion. Bemerkenswerth ist auch hier wieder sein reichliches Auftreten bei dieser verhältnissmässig schwer verdaulichen Eiweisssubstanz, wie beim Eieralbumin, wohingegen es ja bei dem schnell verdauenden Serumalbumin nur in geringen Spuren entsteht. Die peptische Spaltung wird beschleunigt, wenn man vorher das Ammonsulfat durch Dialyse entfernt, wobei die Lösung durch theilweise Abscheidung von Globulin getrübt wird. Das Acidglobulin, das sich nun erst beim Neutralisiren und Aufkochen reichlicher abscheidet, wird während der Verdauung nicht ausgesalzen und die Peptonisirung ist dadurch erleichtert. Auch an diesem Acidglobulin ist eine auffallend starke Kohlenhydratreaction beachtenswerth.

Die Fällungsgrenzen für die Fraction I in neutraler Lösung sind wie auch bei den bisher untersuchten Proteinstoffen 3,8 und 4,6. Sie scheidet sich gut ab in gelben Krusten und weist die Eigenschaften einer primären Albumose auf. $\mathrm{Zu}$ einem geringen Theil besteht sie aus wasserunlöslicher Hetero- 
albumose. Mit Kochsalz wird sie aus ihrer wässerigen Lösung ausgesalzen und zwar bei Sättigung vollkommen. Von einer "umgekehrten Albumosenreaction», die von Kühne und Chittenden an ihrer Protoglobulose constatirt wurde, ist nichts wahrzunehmen. Die Trübung klärt sich beim Erwärmen wie bei den Albumosen. Verdünntes Kupfersulfat, Essigsäure und Ferrocyankalium verursachen eine starke Trübung, dagegen fällt Salpetersäure weder in der Kälte noch in der Wärme. Am stärksten tritt die Reaction auf nichtoxydirten Schwefel hervor, nur sehr gering die Furforolreactionen. Jodquecksilberkalium, Gerbsäure und Trichloressigsäure fällen vollkommen, letztere löst den Niederschlag im Ueberschuss.

Die untere Fällungsgrenze der Fraction II liegt wie die der Albumosen bei 5,6, die obere Grenze dagegen bedeutend höher: bei 7,2 , so dass die Fraction also nicht wie die übrigen aus der Verdauungsflüssigkeit bei Zusatz von $2 / 3$ Volumen, sondern erst bei $3 / 4$ Volumen gesättigter Ammonsulfatiösung vollständig ausgesalzen werden kann. Sie hat eine eigenthümlich klebrige Beschaffenheit und haftet zähe an den Wandungen des Becherglases. Da ihre wässerige Lösung durch verdünntes Kupfersulfat, ferner durch Salpetersäure nicht getrübt wird und nur sehr minimal durch Sättigung mit Kochsalz, hingegen durch Kochsalzsättigung nach Ansäuern vollkommen flockig gefällt wird, dürfen wir sie als Deuteroalbumose ansprechen. Mit Essigsäure und Ferrocyankalium wird sie unvollkommen abgeschieden und gegenüber den Alkaloidreagentien verhält sie sich wie die vorhergehende Fraction. Beim Kochen mit Bleiacetat und Lauge setzt sich wiederum ein sehr reichlicher Niederschlag von Schwefelblei ab, die Millon'sche und die Molisch'sche Reaction ist intensiver geworden.

Die nächste Fraction III, die bei allen anderen Proteinstoffen stets am reichlichsten vorhanden war, ist hier ziemlich spärlich. Ihre Fällungsgrenzen liegen zwischen $7,8-8,6$, so dass sie also durch Sättigung des Verdauungsfiltrates mit gepulvertem Ammoniumsulfat vollkommen ausgeschieden wird. Sie charakterisirt sich ebenfalls als Deuteroalbumose wie die vorhergehende und scheidet sich in gesättigter Kochsalzlösung 
nicht mehr aus. Diese wie alle folgenden Fractionen werden von Trichloressigsäure nicht mehr gefällt. Die Abspaltung von unoxydirtem Schwefel ist nicht mehr so reichlich wie bei den vorausgehenden Fractionen, dagegen tritt jetzt die Furfurolbildung bei der Molisch'schen Probe stärker auf.

Wie beim Eier- und Serumalbumin kann auch hier aus dem salzgesättigten Filtrat durch Zusatz von 1/10 Volumen salzgesättigter Säure oder Ammoniak erwähnter Concentration eine weitere Fraction IV als diffuse weisse Trübung ausgeschieden werden. Sie steht ihren Eigenschaften nach wiederum den Peptonen näher als den Albumosen, indem sie weder durch Kochsalz in saurer Lösung, noch durch Essigsäure und Ferrocyankalium mehr gefällt werden kann. Die Millon'sche sowie die Schwefelreaction fallen sehr schwach aus, dagegen ist eine intensive Molisch'sche Reaction vorhanden.

Fraction V (Pepton A) wird in derselben Weise wie bei den andern Eiweisskörpern als schöner, reichlicher, weisser Alkoholniederschlag gewonnen. Sie ist bei qualitativer Prüfung absolut frei von abspaltbarem Schwefel, die Färbung mit $\alpha$-Naphtol oder Eisessig und concentrirter Schwefelsäure ist höchst intensiv, beim Kochen mit Millon'schem Reagens entsteht schöne Rothfärbung. Gerbsäure fällt einen in der Hitze löslichen, im Säureüberschuss unlöslichen, eigenthümlich bordeauxfarbigen Niederschlag. Trichloressigsäure fällt, wie bemerkt, überhaupt nicht, Jodquecksilberkalium unvollkommen, wobei sich die Trübung in der Hitze wie im Ueberschuss löst.

Die durch Blei vollkommen jodfrei gemachte, in Lösung wasserhelle Fraction VI ist das alkohollösliche Pepton B, das noch sehr intensive rothviolette Biuretreaction und schöne Xanthoproteinreaction ergibt, dagegen keine Spur von unoxydirtem Schwefel mehr erkennen lässt. Auch die Molisch'sche Zuckerprobe ist durchaus negativ. Beim Kochen mit Millon's Reagens entsteht noch schwache Rothgelbfärbung. Von den Alkaloidreagentien fällen Trichloressigsäure und Jodquecksilberkalium überhaupt nicht mehr, Gerbsäure dagegen noch vollständig. 
$\mathrm{Zu}$ Gunsten einer grösseren Uebersichtlichkeit in der Vergleichung der Spaltprodukte verschiedener Abstammung folge auf Seite 298/299 eine kurze tabellarische Zusammenfassung nach ihren hervorstechenden Gruppenreactionen, die auch die hierhergehörigen Erfahrungen E. P. Pick's an den Spaltprodukten des Fibrins ("Witte-Pepton») mitberücksichtigt.

Diese Erfahrungen lehren, dass wir durch die Pepsinspaltung ganz verschiedener Eiweissstoffe, nämlich des Fibrins, Eieralbumins, Serumalbumins und Globulins, eine gleiche Anzahl von Spaltprodukten erhalten, die wir aus den verschiedenen Verdauungslösungen durch fractionirte Fällung mit gesättigter Ammonsulfatlösung darstellen können. Wenngleich auch nicht alle Fractionen Anspruch machen können auf geschlossene chemische Einheitlichkeit - so stellt z. B. die Fraction I ein Gemenge dar von Proto- und Heteroalbumose so liegt doch ein wesentlicher Fortschritt in der schärferen Charakterisirung der einzelnen Componenten, vorzugsweise des Gemenges, das man als Deuteroalbumosen bezeichnet. Gerade hierunter zeichnen sich einzelne, und besonders gilt dies von den Derivaten des krystallinischen Eieralbumins, in ihren äusseren Eigenschaften durch eine so scharf ausgeprägte chemische Individualität aus, dass ihre krystallinische Darstellung, die, wie erwähnt, bei mehr gelegentlichen Versuchen nicht $\mathrm{zu}$ befriedigendem Abschluss führte, sicherlich nur eine Frage der Zeit ist.

Bemerkenswerth ist, dass alle diese Fractionen verschiedenster Provenienz zu ihrer Abscheidung ganz analoger Zusätze concentrirter Ammonsulfatlösung bedürfen; nur die Fraction II (Deuteroalbumose A) des Globulins macht in dieser Hinsicht eine Ausnahme, indem ihre obere ${ }^{1}$ ) Fällungsgrenze gegenüber derjenigen ihrer Schwesterfractionen erheblich in die Höhe gerückt ist.

Hinsichtlich ihrer chemischen Eigenthümlichkeiten besteht unter den entsprechenden Spaltprodukten eine unverkennbare

1) Die unteren Fällungsgrenzen der Fractionen sind weniger massgebend und anscheinend abhängig von dem mehr oder minder reichlichen Vorhandensein der zu fällenden Albumose. 


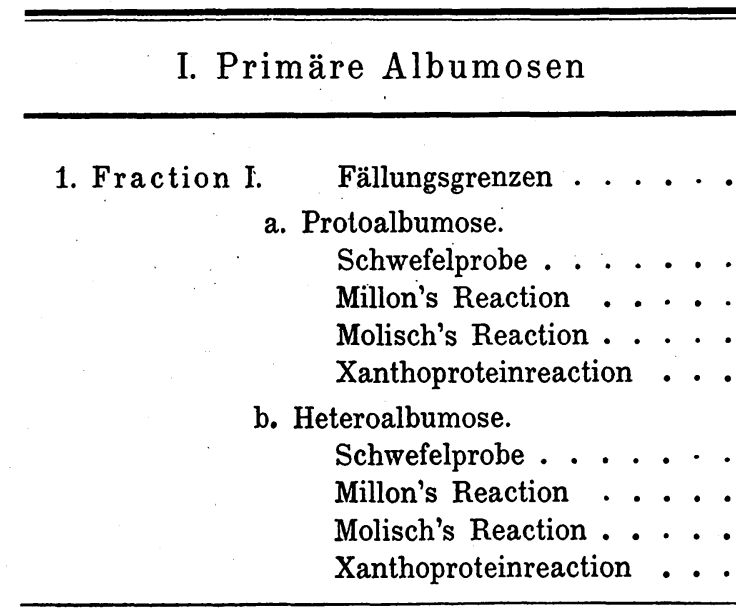

II. Deuteroalbumosen.

2. Fraction II. (Deuteroalbumose A.)

3. Fraction III.
Deuteroalbumose B.)

Fällungsgrenzen .....

Schwefelprobe ......

Millon's Reaction .....

Molisch's Reaction . . . .

Xanthoproteinreaction ...

Fällungsgrenzen . . . .

Schwefelprobe . . . . .

Millon's Reaction . . . .

Molisch's Reaction . . . .

Xanthoproteinreaction ... 4. Fraction IV.
(Deuteroalbumose C.) Fällungsgrenzen ......

Schwefelprobe

Xanthoproteinreaction ...

III. Peptone.

5. Pepton A. (alkoholunlöslich.)

Schwefelprobe

Millon's Reaction .

Molisch's Reaction .....

Xanthoproteinreaction ...

6. Pepton B. (alkohollöslich.)

Schwefelprobe . . . .

Millon's Reaction . . . .

Molisch's Reaction . . . .

Xanthoproteinreaction ..
Fibrin

$2,6-4,4$

mässig positiv positiv

schwach positiv positiv

salzges. Säure $=1 / 10 \mathrm{~V}$ negativ positiv

schwach positiv positiv

\section{negativ}

schwach positiv stark positiv

schwach positiv

negativ

negativ

negativ

negativ 


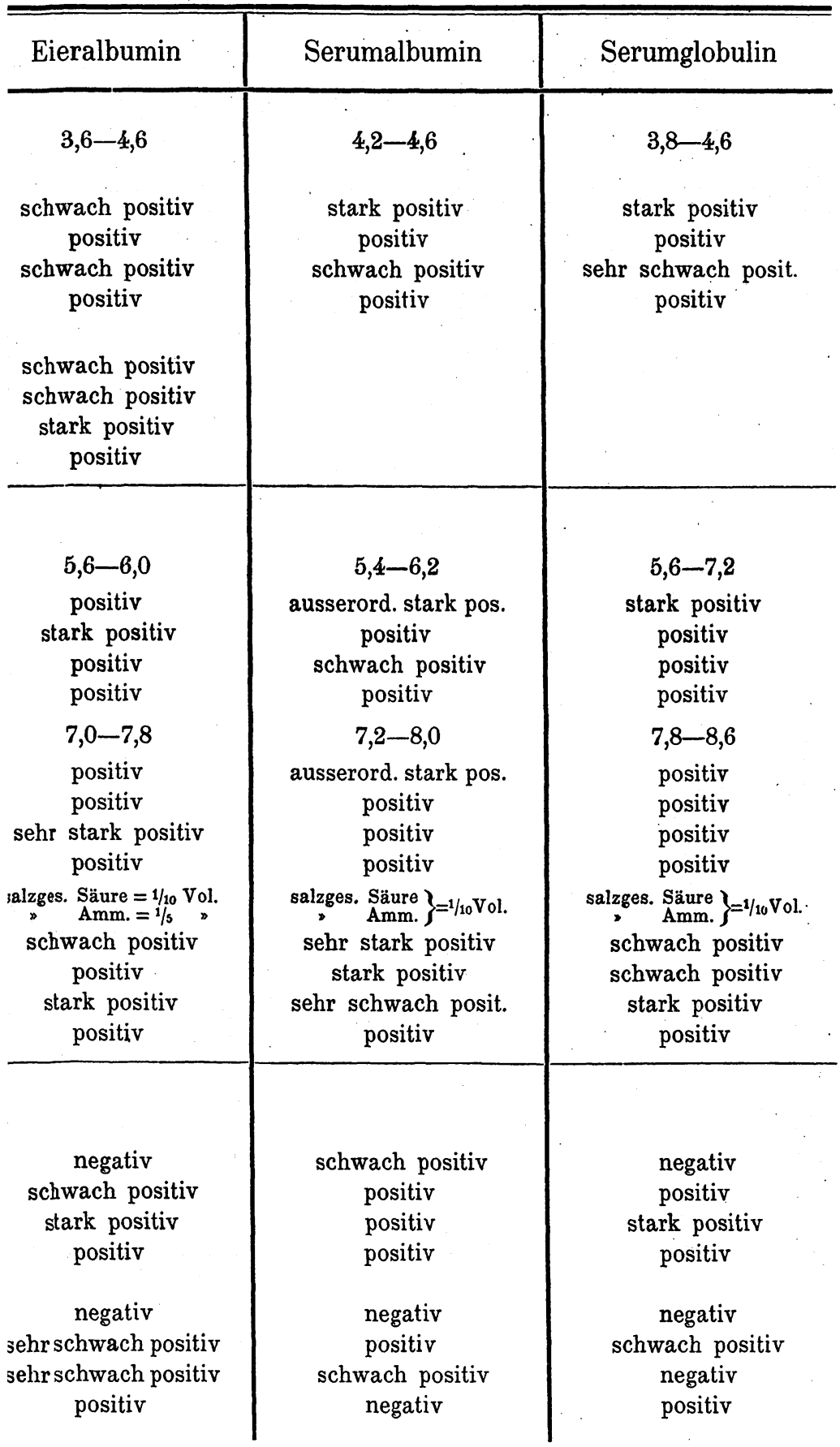


Analogie, so z. B. das allmähliche Verschwinden des unoxydirten Schwefels bei fortschreitender Peptonisirung der Deuteroalbumosen, ferner die augenfällige Zuckerreaction in den Peptonen A u. s. w. Dabei finden geringe Verschiedenheiten bei entsprechenden Fractionen verschiedener Provenienz leicht ihre Erklärung in der Zusammensetzung des Ausgangsmoleküls, so das Hervortreten des abspaltbaren Schwefels in den Fractionen des Serumalbumins, dessen reicher Schwefelgehalt sich zu zwei Dritteln als nichtoxydirter Schwefel durch Kochen mit Blei in alkalischer Lösung abspalten lässt, ${ }^{1}$ ) das Ueberwiegen der Kohlehydratgruppe in den Fractionen des kohlenstoffreichen Eieralbumins ${ }^{2}$ ) u. drgl. m. Die Uebereinstimmung der Eiweissderivate verschiedener Herkunft untereinander weist auf eine nahe Verwandtschaft der Muttercomplexe in den verschiedenen Proteinstoffen hin, deren specifische Eigenthümlichkeiten vielleicht nur durch die Anwesenheit besonderer, dem Molekül angelagerter Gruppen bedingt sind.

Zum Schluss sei noch darauf hingewiesen, dass sich betreffs der Verdaulichkeit der verschiedenen Proteinstoffe bereits bekannte Erfahrungen auch hier insoweit bestätigt haben, als das Serumalbumin am schnellsten, schwerer hingegen das Serumglobulin und am langsamsten das Eieralbumin durch die Pepsinspaltung in Albumosen resp. Peptone übergeführt wurde. So ergab sich für gleichconcentrirte, salzfreie Lösungen der nicht coagulirten Eiweissstoffe, dass das Serumalbumin etwa $2-3$, das Serumglobulin etwa $1 \times 24$, das Eieralbumin etwa $2-3 \times 24$ Stunden bedurfte, damit die Spaltung soweit fortgeschritten war, dass einerseits in der Verdauungsflüssigkeit schon Peptone vorhanden waren, andererseits die ursprünglichen Eiweisse schon in primäre Spaltprodukte übergeführt waren.

1) Schulz, Die Bindungsweise des Schwefels im Eiweiss. Zeitschrift für physiolog. Chemie, Bd. XXIV, 1897.

2) Hofmeister, Ueber jodirtes Eieralbumin. Zeitschr. für phys. Chemie, Bd. XXIV, 1897. 